\title{
PEST INFESTATION IDENTIFICATION IN COCONUT TREES USING DEEP LEARNING
}

\author{
Dr. Abraham Chandy, \\ Associate Professor, \\ Department of Electronics and Communication Engineering, \\ Karunya University, \\ Coimbatore, Tamil Nadu, India. \\ Email: abrahamchandy@,karunya.edu
}

\begin{abstract}
In this paper, we propose a precision agriculture technique to detect various pests in coconut trees with the help of NVIDIA Tegra System on Chip (SoC) along with a camera interfaced drone. The drone flies across the coconut farm and captures the images and processes the data using deep learning algorithm to identify the unhealthy and pest affected trees. The deep learning algorithm uses a set of sample pest database. The Artificial Intelligence (AI) machine learning algorithm is also capable of unsupervised learning from the images that are unstructured. The data is transferred directly to the farmer's smart phone with the help of wi-fi. This helps in timely treatment of pest infected trees and to improve the yield of the trees.
\end{abstract}

Keywords: Artificial Intelligence, Deep Learning, System-on-chip, Pest control, Image processing

\section{INTRODUCTION}

Automatic plant disease identification and recognition tools have proved to be a valuable source of data that assist decision making in farms [1]. Artificial intelligence tools like Deep learning and Convolutional Neural Network $(\mathrm{CNN})$ are gaining popularity in this field as they provide optimum solution for plant disease identification [2]. Earlier, pest detection was done by manual observation. This method is arduous and prone to error. Several plant disease cannot be recognized by bare human eyes. Early disease occurrences are minute in nature. In order to improve the quality of production and yield in plants, it is essential to identify the symptoms in their initial stages and treat the diseases.

Sustainable pest detection techniques should rely on greater depth to gather more valuable information and learn features automatically rather than depending on blind low-level feature descriptors. Since the past two decades, drones are used by farmers to monitor the fields thereby giving way for precision agriculture. Earlier, there used to be a ground control station that is supported by a PC that gathers that data sent by the drone and performs processing of the image and data. With recent developments in technology, the processing of the

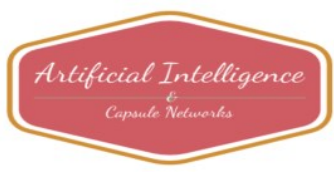


Journal of Artificial Intelligence and Capsule Networks (2019)

Vol.01/ No. 01

Pages: $10-18$

http://irojournals.com/aicn/

DOI: https://doi.org/10.36548/jaicn.2019.1.002

images can be done in the drone itself and the processed data is directly sent to the farmer's smart device with a user friendly interface.

Coconut trees or Cocos nucifera are versatile and have numerous uses. Pest infestation in coconut trees have always been a challenge due to height of the trees and complexity in identification. In this paper, we develop a method to identify the pests in coconut trees with the help of a drone [3] to fly across the field and capture images of the trees, process the images and identify pest infestation via image processing and deep learning. Further, the information is sent to the farmer's smart phone with the help of Wifi.

Some of the most common types of pest infestation and diseases in coconut trees are tabulated along with their symptoms and causes.

Table 1: Pest infestation and diseases in coconut trees

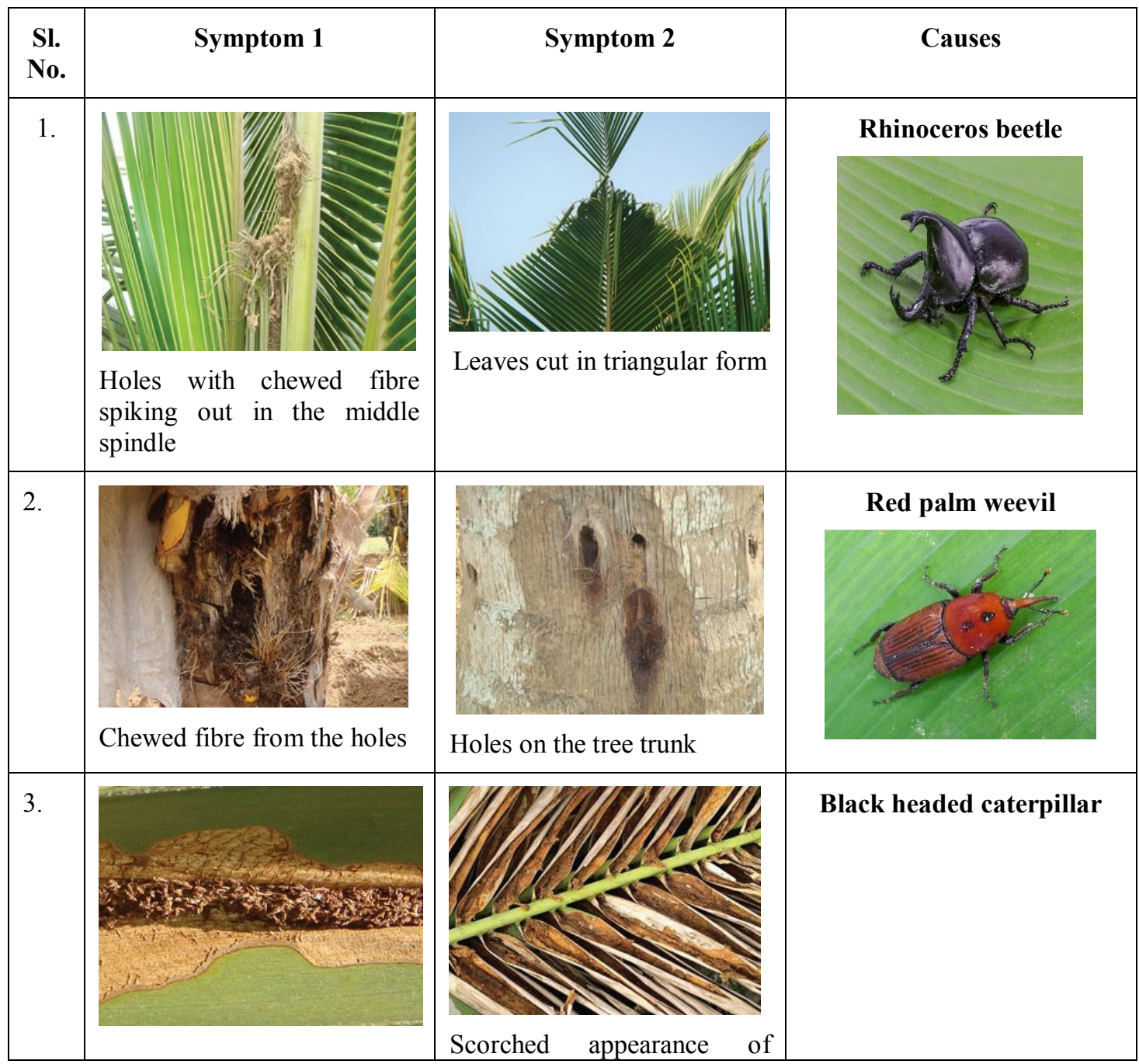


Journal of Artificial Intelligence and Capsule Networks (2019)

Vol.01/ No. 01

Pages: $10-18$

http://irojournals.com/aicn/

DOI: https://doi.org/10.36548/jaicn.2019.1.002

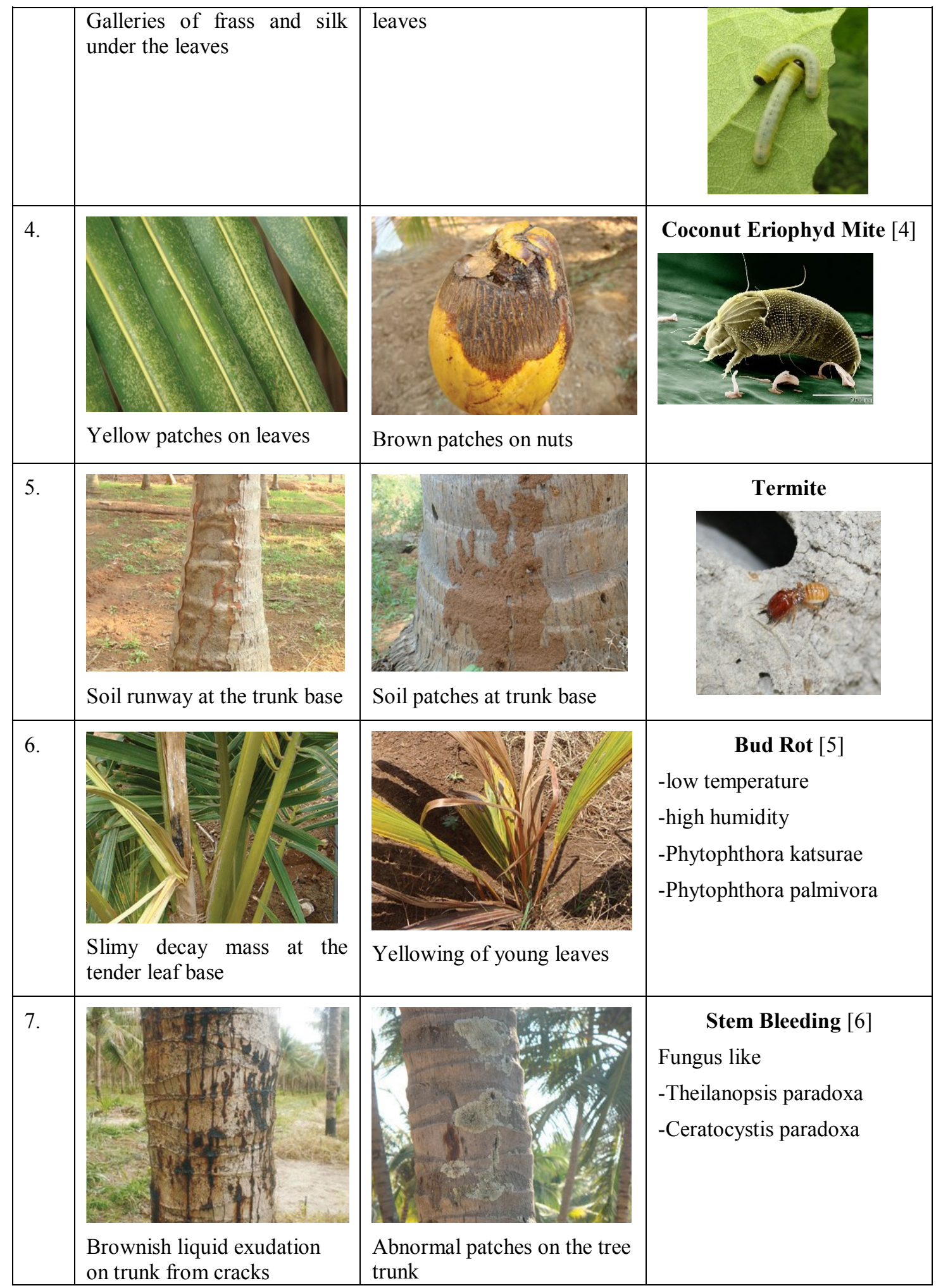

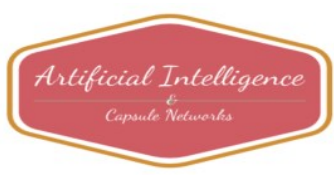




\begin{tabular}{|c|c|c|c|}
\hline 8. & 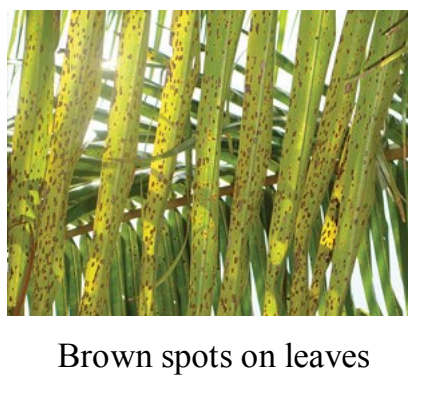 & $\begin{array}{l}\text { Leaves enlarge in fanlike } \\
\text { structure and end up rotting }\end{array}$ & $\begin{array}{l}\quad \text { Leaf Rot [7] } \\
\text { Fungus like } \\
\text {-Exserohilum rostratum } \\
\text {-Colletotrichum } \\
\text { gloeosporioides } \\
\text {-Fusarium spp }\end{array}$ \\
\hline 9. & $\begin{array}{l}\text { P } \\
\text { Pollen sterility and shedding } \\
\text { of immature nuts }\end{array}$ & $\begin{array}{c}\text { Abnormal inward bending of } \\
\text { leaflets }\end{array}$ & $\begin{array}{l}\quad \text { Root Wilt }[8] \\
\text {-Mycoplasma Like Organism } \\
\text {-Lace wing bug } \\
\text {-Plant hopper } \\
\text {-Stephanitis typicus } \\
\text {-Proutista moesta }\end{array}$ \\
\hline
\end{tabular}

\section{EXISTING LITERATURE}

Izzeddin et al. [9] developed an expert system for diagnosis of diseases in coconut tree using CLIPS and Delphi object expert system language. The expert system contains a computer application based on artificial intelligence (AI) that has a knowledge base along with an inference engine. Jayme et al. [10], proposed a system for identifying abrasions and spots with the help of deep learning techniques. They also identified that the limitation of dataset in terms of number of samples and variety of symptoms, inhibit the development of comprehensive systems and proposed a solution to increase the image dataset size as well as the diversity of data.

Image segmentation with HSI algorithm can be used to classify various plant diseases [11]. Genetic algorithms are very efficient in image segmentation. In this technique, the plant images are captured with a digital camera and processed using image enhancement techniques. Further, the unwanted area is clipped and smoothing algorithm is applied. Based on the colour variations from pixel to pixel and the intensity of green colour, the leaves are considered healthy or unhealthy. Liu et al. [12], use Position-Sensitive Score Map (PSSM) for classifying the pest and Contextual Region of Interest due to its accuracy in pest detection. The system

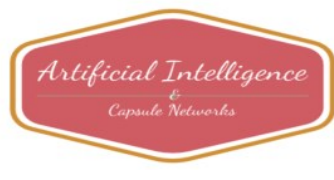


Journal of Artificial Intelligence and Capsule Networks (2019)

Vol.01/ No. 01

Pages: $10-18$

http://irojournals.com/aicn/

DOI: https://doi.org/10.36548/jaicn.2019.1.002

provided 75.46\% mean Average Precision. Pablo et al. [13], developed a convoy of diverse ground and aerial robots equipped with advanced sensors, improved end effectors and enhanced decision control algorithms to cover a huge range of agricultural circumstances.

Machine Learning and Artificial Intelligence are applied in agriculture for several purposes. IoT in combination with machine learning can also help control of farm equipment thereby creating a smart farm environment. The flexibility of manual control based on the processed data received will be of great help to the farmers to even remotely access the farm equipment. Karandeep Kaur in his paper has provided a brief of various machine learning algorithm used in agriculture. As shown in the table below

Table 2: Machine Learning Algorithm in Agriculture [14]

\begin{tabular}{|l|l|l|l|}
\hline \multirow{2}{*}{$\begin{array}{c}\text { Sl. } \\
\text { No. }\end{array}$} & \multicolumn{3}{|c|}{ Machine Learning Applications in Farming } \\
\cline { 2 - 4 } & \multicolumn{1}{|c|}{ Field of Study } & \multicolumn{1}{c|}{ Author } & \multicolumn{1}{c|}{ Algorithm } \\
\hline 1 & $\begin{array}{l}\text { Crop selection, } \\
\text { Crop yield prediction }\end{array}$ & $\begin{array}{l}\text { Washington et al, } \\
\text { Shivnath et al, } \\
\text { Snehal et al, } \\
\text { Rakesh et al }\end{array}$ & $\begin{array}{l}\text { Classification algorithm, } \\
\text { Back propagation neural } \\
\text { network, Neural } \\
\text { Network, CSM }\end{array}$ \\
\hline 2 & Weather Forecasting & Radhika et al & Support Vector Machine \\
\hline 3 & Smart Irrigation System & Aditya Gupta et al & General ML algorithm \\
\hline 4 & Crop disease prediction & $\begin{array}{l}\text { Rumpf et al, Raj et al, Mehra } \\
\text { et al }\end{array}$ & $\begin{array}{l}\text { Support vector machines, } \\
\text { pattern recognition, } \\
\text { ANN, regression trees, } \\
\text { random forests }\end{array}$ \\
\hline 5 & Deciding the MSP & Karandeep Kaur & $\begin{array}{l}\text { Classification } \\
\text { techniques, Neural } \\
\text { networks etc. }\end{array}$ \\
\hline
\end{tabular}

In summary, some of the major methods used for plant disease detection and analysis are Back propagation neural network, Combining morphological features of leaves, feed-forward neural network based classifier, image processing, wavelet based image processing and neural network, Probabilistic neural network, Zooming algorithm, Self-organizing map, Image clipping, Otsu segmentation and so on [15]. AI also imposes certain challenges in implementation. Training different models require high quality image data for the generation of accurate outputs. In case of drones, operational safety, privacy concerns and insurance protection are of concern.

\section{PROPOSED WORK}

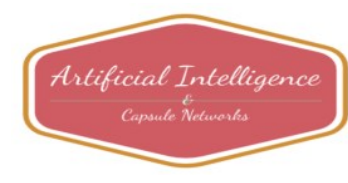




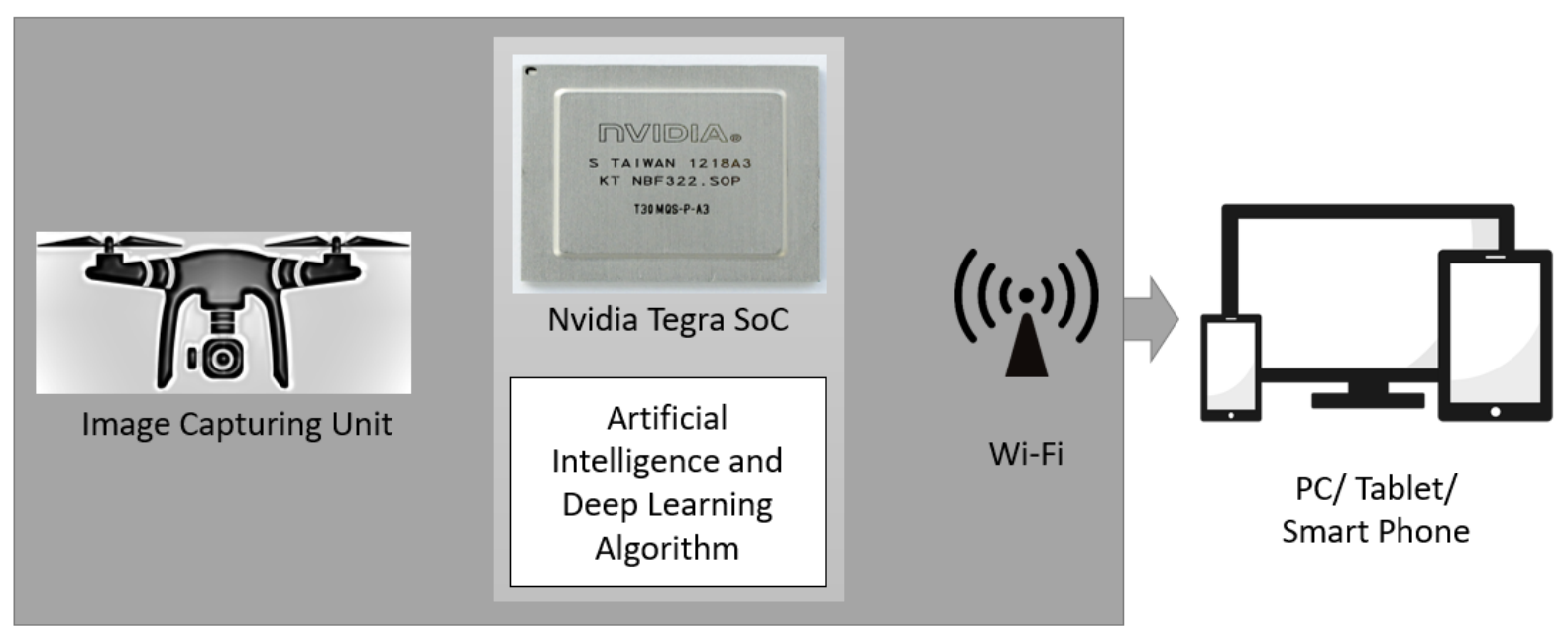

Figure 1 Block diagram of pest detection system

In this system, image classification is done using deep learning as it allows storing the image database to represent a wide variety of symptoms and conditions that are found in the affected coconut trees. Data augmentation is done with the new samples collected from the coconut trees. We design an image capturing unit that is connected to a quad-copter. The drones used for agriculture must strictly follow the regulation of not flying within 5 miles of airports, flying under a height of 400 feet, being in the operator's line of sight and respect the public privacy. We mount a simple GoPro camera for the image capturing purpose.

The features are extracted from the original images and learning is done. No pre-processing is required for this system. Nvidia Tegra [16] SoC with ARM Cortex based CPU and GPU is also embedded in the drone for processing the images. The system is power efficient and supports machine learning applications. It can also support high definition still camera and camcorder functions. It is a Computer Unified Device Architecture (CUDA) supported device. The deep neural networks running on the device has better privacy, inbuilt memory and low energy consumption.

We build a dataset with the sample images of pests and infected coconut tree segments like trunk, leaves and nuts. The images are annotated with labels by agricultural experts. Channel-spatial attention (CSA) and Region proposal network (RPN) [17] is used to distinguish between the pest affected and normal regions as well as indicate the likely pest position. Speed and accuracy of image capture and processing is made possible due to the $\mathrm{SoC}$ and fast interpreting algorithm. Based on the image, the severity of the disease can also be identified. The image is segmented into pixels and based on the colour information from the pixels, the level of health of the leaves are identified and analysed.

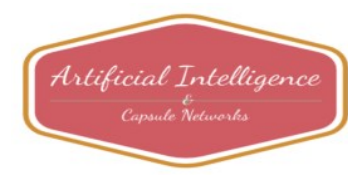


Pages: $10-18$

http://irojournals.com/aicn/

DOI: https://doi.org/10.36548/jaicn.2019.1.002

The processed data is matched with a deep learning data sets to identify the type of disease and type of pesticide to be used. The information along with the location of the pest, type of disease, image, area of infestation and optimal pesticide is sent to the farmer's smart device with the help of Wi-Fi directly from the drone. We use ESP8266 Wi-Fi module for data transfer. It can send 64 character data strings. Based on the requirement, more advanced and high data rate Wi-Fi modules can also be used in the system. A smart and user friendly interface is also created for the farmer to access the data and required specifications as per his interest. In order to verify the efficiency of this system, the most frequently identified samples are kept in the cache memory for faster identification.

\section{RESULT}

We have developed a smart drone that captures images from coconut trees in a prefixed farm area and compares the data with a dataset of pests and diseases. We deal with a total of 5 pest attacks and 4 plant diseases. Images that show infested and infected areas, are processed using artificial intelligence and deep learning algorithm in the Nvidia Tegra SoC. Information such as pest infestation, yield prediction, precision fertilizer, precision irrigation and so on are provided. Information such as moisture and $\mathrm{pH}$ of the farm land also can be gathered. Further, this information is broadcasted to the agriculturist's smart device with the help of an android interface or application through Wi-Fi.

\section{CONCLUSION AND FUTURE SCOPE}

The future of farming is dependent on adapting cognitive solutions. Even though research is still being carried out in improving the application of artificial intelligence in farming, various applications are already existing and in use. In our system, the drone collects various crop specific data pertaining to coconut trees. This data can be used for analysing the time taken for the trees and nuts to mature and drafting prevention schemes from common pest attacks. Future scope involves making the drone capable to carry pesticides to the top of the trees and spray them directly in the pest affected areas. Also, a system to automatically pluck the ripe coconuts can also be of great use to the agriculturists. Also, the farmer will be able to instruct the drone on specific operations manually, if required. Storage of this data in cloud and creating an IoT hub for interconnection of all the farm equipment is also proposed as future work.

\section{References}


Journal of Artificial Intelligence and Capsule Networks (2019)

Vol.01/ No. 01

Pages: $10-18$

http://irojournals.com/aicn/

DOI: https://doi.org/10.36548/jaicn.2019.1.002

[1] Noguchi, N., J. F. Reid, Q. Zhang, and L. F. Tian. "Vision intelligence for precision farming using fuzzy logic optimized genetic algorithm and artificial neural network." American Society of Agricultural Engineers, St. Joseph, MI, ASAE paper 983034 (1998).

[2] Jhuria, Monika, Ashwani Kumar, and Rushikesh Borse. "Image processing for smart farming: Detection of disease and fruit grading." In 2013 IEEE Second International Conference on Image Information Processing (ICIIP-2013), pp. 521-526. IEEE, 2013.

[3] Puri, Vikram, Anand Nayyar, and Linesh Raja. "Agriculture drones: A modern breakthrough in precision agriculture." Journal of Statistics and Management Systems 20, no. 4 (2017): 507-518.

[4] Sathiamma, B., CP Radhakrishnan Nair, and P. K. Koshy. "Outbreak of a nut infesting eriophyid mite Eriophyes guerreronis (K.) in coconut plantations in India." Indian Coconut Journal (Cochin) 29, no. 2 (1998): $1-3$.

[5] Butler, E. J. "Bud-rot of Coconut and other Palms." Bud-rot of Coconut and other Palms. (1925).

[6] Warwick, Dulce, and Edson EM Passos. "Outbreak of stem bleeding in coconuts caused by Thielaviopsis paradoxa in Sergipe, Brazil." Tropical Plant Pathology 34, no. 3 (2009): 175-177.

[7] Srinivasan, N., and M. Gunasekaran. "Incidence of fungal species associated with leaf rot disease of coconut palms in relation to weather and the stage of lesion development." Annals of applied biology129, no. 3 (1996): 433-449.

[8] Manimekalai, R., V. P. Soumya, R. Sathish Kumar, R. Selvarajan, K. Reddy, G. V. Thomas, M. Sasikala, G. Rajeev, and V. K. Baranwal. "Molecular detection of 16SrXI group phytoplasma associated with root (wilt) disease of coconut (Cocos nucifera) in India." Plant disease 94, no. 5 (2010): 636-636

[9] Alshawwa, Izzeddin A., Abeer A. Elsharif, and Samy S. Abu-Naser. "An Expert System for Coconut Diseases Diagnosis." (2019). 
Journal of Artificial Intelligence and Capsule Networks (2019)

Vol.01/ No. 01

Pages: $10-18$

http://irojournals.com/aicn/

DOI: https://doi.org/10.36548/jaicn.2019.1.002

[10] Barbedo, Jayme Garcia Arnal. "Plant disease identification from individual lesions and spots using deep learning." Biosystems Engineering 180 (2019): 96-107.

[11] Reddy, N. Hanuman, E. Ravi Kumar, M. Vinay Reddy, KL Raghavender Reddy, and G. Susmitha Valli. "Bioinformatics and image processing — detection of plant diseases." In First International Conference on Artificial Intelligence and Cognitive Computing, pp. 149-154. Springer, Singapore, 2019.

[12] Liu, Liu, Rujing Wang, Chengjun Xie, Po Yang, Fangyuan Wang, Sud Sudirman, and Wancai Liu. "PestNet: An End-to-End Deep Learning Approach for Large-Scale Multi-Class Pest Detection and Classification." IEEE Access 7 (2019): 45301-45312.

[13] Gonzalez-de-Santos, Pablo, Angela Ribeiro, Cesar Fernandez-Quintanilla, Francisca Lopez-Granados, Michael Brandstoetter, Slobodanka Tomic, Stefania Pedrazzi et al. "Fleets of robots for environmentally-safe pest control in agriculture." Precision Agriculture 18, no. 4 (2017): 574-614.

[14] Kaur, Karandeep. "Machine learning: applications in Indian agriculture." International Journal of Advanced Research in Computer and Communication Engineering 5, no. 4 (2016): 342-344.

[15] Patil, Jayamala K., and Raj Kumar. "Advances in image processing for detection of plant diseases." Journal of Advanced Bioinformatics Applications and Research 2, no. 2 (2011): 135-141.

[16] Ditty, Michael, Tegra Architecture, John Montrym, and Craig Wittenbrink. "NVIDIA's Tegra K1 systemon-chip." In 2014 IEEE Hot Chips 26 Symposium (HCS), pp. 1-26. IEEE, 2014.

[17] Ren, Shaoqing, Kaiming He, Ross Girshick, and Jian Sun. "Faster r-cnn: Towards real-time object detection with region proposal networks." In Advances in neural information processing systems, pp. 91-99. 2015 .

[18] Stavrakoudis, Dimitris, Dimitrios Katsantonis, Kalliopi Kadoglidou, Argyris Kalaitzidis, and Ioannis Z. Gitas. "Estimating Rice Agronomic Traits Using Drone-Collected Multispectral Imagery." Remote Sensing 11, no. 5 (2019): 545 . 
Journal of Artificial Intelligence and Capsule Networks (2019)

Vol.01/ No. 01

Pages: $10-18$

http://irojournals.com/aicn/

DOI: https://doi.org/10.36548/jaicn.2019.1.002

[19] Stehr, Nikki J. "Drones: The newest technology for precision agriculture." Natural Sciences Education 44, no. 1 (2015): 89-91.

[20] Dharmaraj, V., and C. Vijayanand. "Artificial Intelligence (AI) in Agriculture." Int. J. Curr. Microbiol. App. Sci 7, no. 12 (2018): 2122-2128.

[21] Chandra, Ranveer. "FarmBeats: AI \& IoT for Agriculture." (2017).

[22] Ryu, Minwoo, Jaeseok Yun, Ting Miao, Il-Yeup Ahn, Sung-Chan Choi, and Jaeho Kim. "Design and implementation of a connected farm for smart farming system." In 2015 IEEE SENSORS, pp. 1-4. IEEE, 2015.

[23] Tripicchio, Paolo, Massimo Satler, Giacomo Dabisias, Emanuele Ruffaldi, and Carlo Alberto Avizzano. "Towards smart farming and sustainable agriculture with drones." In 2015 International Conference on Intelligent Environments, pp. 140-143. IEEE, 2015. 